OPEN ACCESS

Edited by:

Craig Atwood,

University of Wisconsin-Madison,

United States

Reviewed by:

Xudong Huang,

Massachusetts General Hospital,

Harvard Medical School,

United States

Boon-Seng Wong,

Singapore Institute of Technology,

Singapore

*Correspondence:

Zumin Shi

zumin@qu.edu.qa

Received: 25 January 2020 Accepted: 26 March 2020

Published: 15 April 2020

Citation:

Al-Ghazali K, Eltayeb S, Musleh A,

Al-Abdi T, Ganji V and Shi Z

(2020) Serum Magnesium and

Cognitive Function Among

Qatari Adults.

Front. Aging Neurosci. 12:101. doi: 10.3389/fnagi.2020.00101

\section{Serum Magnesium and Cognitive Function Among Qatari Adults}

\author{
Kateba Al-Ghazali, Sana Eltayeb, Ayesha Musleh, Tamara Al-Abdi, Vijay Ganji \\ and Zumin Shi* \\ Human Nutrition Department, College of Health Sciences, QU Health, Qatar University, Doha, Qatar
}

Background: Previous studies found that low blood magnesium increases the risk of several diseases such as cardiovascular diseases (CVD), diabetes, and hypertension. These ailments are associated with declined cognitive function.

Objective: We aimed to examine the association between serum magnesium and cognitive function among Qatari adults. In addition, we assessed the interaction relation between low serum magnesium, hypertension, and diabetes in relation to cognitive function.

Method: Data from 1,000 Qatari participants aged $\geq 20$ years old who participated in the Qatar Biobank (QBB) Study were analyzed. Serum magnesium was measured by an automated calorimetric method and suboptimal magnesium was characterized by $<0.85 \mathrm{mmol} / \mathrm{l}$. Cognitive function was determined by measuring the mean reaction time (MRT) based on a computer-based, self-administered test. Multivariable linear regression was used to examine the relation between serum magnesium concentrations and cognitive function.

Results: The prevalence of suboptimal magnesium was $57.1 \%$. Across the four quartiles of serum magnesium from the lowest to the highest, the regression coefficients $(95 \% \mathrm{Cl})$ for MRT were 0 (reference), $-17.8,-18.3$, and -31.9 (95\% Cl 2.4-3.1; $p$ for trend 0.05). The presence of hypertension and diabetes significantly increased the MRT. Women with suboptimal magnesium and hypertension had the highest MRT.

Conclusion: The prevalence of suboptimal magnesium is high in Qatar. There was a direct association between serum magnesium and cognitive function. Low magnesium concentrations were associated with a longer MRT.

Keywords: serum magnesium, mean reaction time, cognition, qatar biobank, adults, cross-sectional study

\section{INTRODUCTION}

Dementia is a major neurocognitive disorder characterized by a decline in memory, problemsolving, language, and other cognitive skills (Hugo and Ganguli, 2014). According to the World Alzheimer Report 2018, 50 million people are living with dementia and it will triple to 152 million by 2050 (Alzheimer's Disase International, 2018). Nutrition is an important modifiable factor that affects cognitive function (Scarmeas et al., 2018). Most of the existing studies found a beneficial effect of fish, nuts, and fresh vegetable intake on cognition (Scarmeas et al., 2018). However, the association between individual nutrients intake and cognition is inconclusive (Scarmeas et al., 2018). 
Magnesium is one of the most abundant cations in the human body (Jahnen-Dechent and Ketteler, 2012). It is a cofactor for more than 300 enzymatic reactions. These enzymes function in regulating blood pressure, controlling blood glucose, and synthesis of several biomolecules such as proteins, DNA, and RNA (Gröber et al., 2015). Moreover, magnesium plays a role in neuromuscular conduction and nerve-impulse transmission. It also has a protective role against neuronal cell death (Kirkland et al., 2018). Therefore, magnesium may have a potential role in neurological disorders (Kirkland et al., 2018).

Magnesium can be found in various food sources such as nuts, legumes, dairy products, and green leafy vegetables. In modern society with a high consumption of refined grains and low intake of vegetables, the intake of magnesium is low (Costello R. et al., 2016). Serum concentrations of magnesium is used as a marker of magnesium nutritional status. Studies have shown that low magnesium can increase the risk of several diseases such as cardiovascular diseases (CVD; Fang et al., 2016; Azab et al., 2019), diabetes mellitus, and hypertension (Costello R. et al., 2016). These diseases are associated with declined cognitive function (Biessels and Despa, 2018).

Evidence from animal studies supports the role of magnesium in cognitive function. For example, a study conducted by $\mathrm{Xu}$ et al. (2014) found that magnesium protects cognitive function and synaptic plasticity by inhibiting GSK-3 $\beta$ in a rat model with Alzheimer's disease. However, human studies on magnesium and cognition are scarce across the world. Higher dietary magnesium intake was associated with a reduced risk of developing cognitive impairment in an 8-year follow-up study in Australia (Cherbuin et al., 2014). The findings are supported by the fact that Alzheimer's disease patients have lower plasma magnesium concentrations (Barbagallo et al., 2011). In stroke patients, low serum magnesium concentrations were associated with post-stroke cognitive impairment (Tu et al., 2018). However, the relation between magnesium and cognitive function in the general population is not clear.

A recent study on 9,693 Qatari adults found that subclinical magnesium deficiency (serum magnesium $<0.85 \mathrm{mmol} / \mathrm{l}$ ) is common. Low circulating magnesium is positively associated with prediabetes, diabetes, and hypertension in Qatari adults (Shi and Abou-Samra, 2019). However, no studies have assessed the relationship between serum magnesium and cognition in Qatar, where the prevalence of diabetes is about $\sim 19 \%$ (AlThani et al., 2017a). Therefore the objectives of this study were to determine the association between serum magnesium and cognitive function and to assess the interaction relation between low serum magnesium, hypertension, and diabetes in relation to cognitive function in Qatari adults.

\section{MATERIALS AND METHODS}

\section{Study Population}

Adults aged 20 years and above were recruited by Qatar Biobank (QBB). They were Qatari nationals or were living in the country for more than 15 years. These subjects were followed up for every 5 years. The details of the study design were published elsewhere (Al Kuwari et al., 2015).
Sociodemographic information, lifestyle factors, and dietary habits were collected using a self-administered questionnaire. Whereas a nurse interview was used to collect information on health conditions, family history of the disease, and medication use. Each participant underwent a health examination at Hamad Medical Center, Doha.

Body height and weight were measured using a Seca stadiometer. Blood samples $(60 \mathrm{ml})$ were taken and measured for a total of 66 biomarkers at the QBB facility. A random sample of 1,000 Qatari participants aged 20 years and older were included in the current analysis. All the participants had measured serum magnesium concentration and underwent a cognitive function test.

\section{Outcome Variable: Cognitive Function (Mean Reaction Time)}

Mean reaction time (MRT) was used as an indicator of cognitive function. It was measured by a computer-based, self-administered touch screen test comprising 60 tasks (Al Kuwari et al., 2015; Lyall et al., 2016). The stimulus used for the assessment of MRT was visual. In addition to MRT, a paired episodic memory test was conducted. However, due to the limited variation of the test results, the memory test was not included in the current study.

\section{Exposure Variable: Serum Magnesium}

Serum Magnesium was assessed by an automated colorimetric method (Magnesium Gen.2 from Roche Diagnostics, Indianapolis, IN, USA) in the central lab of QBB (Costello R. B. et al., 2016). The coefficients of variations are $0.3-0.8 \%$. Subclinical magnesium deficiency was characterized as serum magnesium $<0.85 \mathrm{mmol} / \mathrm{l}$.

\section{Covariates}

Gender, age, education (low: without university degree; high: with a university degree), smoking (non-smokers, ex-smokers, and current smokers), leisure-time physical activity level [the metabolic equivalent of task (MET), recoded as tertiles], and BMI (overweight was defined as a BMI of 25.0-29.9 kg/m² and obesity was defined as a BMI of $\geq 30 \mathrm{~kg} / \mathrm{m}^{2}$; Al-Thani et al., 2019) were used as covariates. The diagnostic criteria for assessing diabetes were: $\mathrm{HbA} 1 \mathrm{c} \geq 6.5 \%$, random blood glucose $\geq 11.1 \mathrm{mmol} / \mathrm{l}$, fasting blood glucose $\geq 7 \mathrm{mmol} / 1$, or self-reported diabetes (American Diabetes Association, 2014). Hypertension was diagnosed as systolic blood pressure $>140 \mathrm{mmHg}$ and/or diastolic blood pressure $>90 \mathrm{mmHg}$ or previous doctor diagnosis.

\section{Data Analysis}

The serum magnesium concentrations were stratified into gender-specific quartiles. Mean (SD) or percentage were used to present the sample characteristics. To compare the differences between quartile sample characteristics, a Chi-squared test was used for categorical variables and ANOVA was used for continuous variables. To assess the association between serum magnesium and MRT, three multivariable linear regression models were used. Model 1 was adjusted for gender and age (continuous), Model 2 was further 
TABLE 1 | Sample characteristics by quartiles of serum magnesium concentrations ${ }^{1}$.

\begin{tabular}{|c|c|c|c|c|c|}
\hline & $\begin{array}{c}\text { Q1 } \\
N=294\end{array}$ & $\begin{array}{c}\text { Q2 } \\
N=242\end{array}$ & $\begin{array}{c}\text { Q3 } \\
N=237\end{array}$ & $\begin{array}{c}\text { Q4 } \\
N=227\end{array}$ & $p$-value \\
\hline Magnesium (mmol/l) & $0.77(0.03)$ & $0.82(0.01)$ & $0.86(0.01)$ & $0.91(0.03)$ & $<0.001$ \\
\hline Age (years) & 36.1 (10.3) & $35.3(10.3)$ & $35.9(10.7)$ & $36.1(10.0)$ & 0.80 \\
\hline Gender & & & & & 0.24 \\
\hline Male & 152 (51.7\%) & $113(46.7 \%)$ & $129(54.4 \%)$ & 106 (46.7\%) & \\
\hline Female & 142 (48.3\%) & $129(53.3 \%)$ & 108 (45.6\%) & $121(53.3 \%)$ & \\
\hline Education & & & & & 0.32 \\
\hline Low & 105 (35.7\%) & 73 (30.3\%) & 88 (37.3\%) & 72 (31.7\%) & \\
\hline High & 189 (64.3\%) & 168 (69.7\%) & 148 (62.7\%) & 155 (68.3\%) & \\
\hline Smoking status & & & & & 0.56 \\
\hline Non-smoker & 192 (65.3\%) & $161(66.5 \%)$ & 158 (66.7\%) & 162 (71.4\%) & \\
\hline Current Smoker & 53 (18.0\%) & 45 (18.6\%) & 48 (20.3\%) & $41(18.1 \%)$ & \\
\hline Ex-smoker & 49 (16.7\%) & 36 (14.9\%) & $31(13.1 \%)$ & 24 (10.6\%) & \\
\hline Leisure time physical activity (MET h/week) & 7.9 (32.3) & $4.5(12.8)$ & $7.0(20.6)$ & $5.2(16.4)$ & 0.30 \\
\hline $\mathrm{BMI}\left(\mathrm{kg} / \mathrm{m}^{2}\right)$ & $28.8(5.5)$ & $28.3(5.7)$ & $27.6(5.9)$ & $28.0(5.8)$ & 0.11 \\
\hline BMI categories & & & & & 0.084 \\
\hline Normal & 69 (23.5\%) & $71(29.3 \%)$ & 85 (35.9\%) & $68(30.0 \%)$ & \\
\hline Overweight & $120(40.8 \%)$ & $87(36.0 \%)$ & $86(36.3 \%)$ & $89(39.2 \%)$ & \\
\hline Obese & $105(35.7 \%)$ & 84 (34.7\%) & $66(27.8 \%)$ & $70(30.8 \%)$ & \\
\hline Supplement use & $188(63.9 \%)$ & $143(59.1 \%)$ & $145(61.2 \%)$ & $138(60.8 \%)$ & 0.71 \\
\hline Vitamin D and Calcium supplements use & $126(42.9 \%)$ & $93(38.4 \%)$ & $84(35.4 \%)$ & $80(35.2 \%)$ & 0.23 \\
\hline $\mathrm{HbA1C}(\%)$ & $5.7(1.3)$ & $5.5(0.8)$ & $5.5(0.7)$ & $5.5(0.6)$ & 0.002 \\
\hline Hypertension & $33(11.2 \%)$ & $18(7.4 \%)$ & $24(10.1 \%)$ & $21(9.3 \%)$ & 0.51 \\
\hline Diabetes & $46(16.2 \%)$ & $27(11.6 \%)$ & $21(9.2 \%)$ & $22(10.2 \%)$ & 0.069 \\
\hline Insulin use & $13(4.4 \%)$ & $3(1.2 \%)$ & $3(1.3 \%)$ & $0(0.0 \%)$ & 0.001 \\
\hline Diabetes medication use other than insulin & $29(9.9 \%)$ & $10(4.1 \%)$ & $8(3.4 \%)$ & $8(3.5 \%)$ & 0.002 \\
\hline Hypertension medication use & $26(8.8 \%)$ & $10(4.1 \%)$ & $10(4.2 \%)$ & $9(4.0 \%)$ & 0.030 \\
\hline Mean reaction time (millisecond) & $732(218)$ & $710(198)$ & $711(215)$ & $703(178)$ & 0.38 \\
\hline
\end{tabular}

${ }^{1}$ Values are mean (SD) or $n(\%)$.

TABLE 2 | Association between serum magnesium concentrations and cognitive function ${ }^{1}$.

\begin{tabular}{|c|c|c|c|c|c|c|c|}
\hline & \multicolumn{5}{|c|}{ Quartiles of serum magnesium } & \multirow[b]{2}{*}{ Magnesium (continuous) } & \multirow[b]{2}{*}{$p$} \\
\hline & Q1 & Q2 & Q3 & Q4 & $p$ for trend & & \\
\hline Model $1^{2}$ & 0 (reference) & $-19.9(-50.9$ to 11.1$)$ & $-16.4(-47.5$ to 14.8$)$ & $-34.1(-65.6$ to -2.54$)$ & 0.049 & $-198.9(-391.4$ to -6.3$)$ & 0.043 \\
\hline Model $2^{3}$ & 0 (reference) & $-17.8(-48.4$ to 12.8$)$ & $-18.3(-49.0$ to 12.5$)$ & $-31.9(-63.0$ to -0.82$)$ & 0.05 & $-206.3(-396.1$ to -16.4$)$ & 0.033 \\
\hline Model $3^{4}$ & 0 (reference) & $-9.3(-40.4$ to 21.8$)$ & $-10.7(-42.2$ to 20.8$)$ & $-24.7(-56.5$ to 7.2$)$ & 0.14 & $-140.1(-336.2$ to 56.1$)$ & 0.16 \\
\hline
\end{tabular}

${ }^{1}$ Values are regression coefficients (95\% Cl) from multivariable linear regression. ${ }^{2}$ Adjusted for age and gender. ${ }^{3}$ Further adjusted for education, smoking, and physical activity. ${ }^{4}$ Further adjusted for BMI, diabetes, hypertension, and medication for diabetes and hypertension. $p$ values $<0.05$.

adjusted for smoking, education, BMI, and physical activity. Model 3 was further adjusted hypertension, diabetes and the for use of medication for hypertension and diabetes. The interaction between serum magnesium, cognitive function, and chronic diseases (diabetes and hypertension) was tested by adding the product terms of the variables in the linear regression model. To visualize the interaction, a command (marginsplot) in Stata was used. All the analyses were conducted using STATA (Version 16, Stata Corporation, College Station, TX, USA). We considered $p$-values $<0.05$ (2-tailed) as statistically significant.

\section{RESULTS}

\section{Sample Description}

The mean age of the sample was 35.8 (SD 10.3) years. The mean serum magnesium concentration was 0.84 (SD 0.08) $\mathrm{mmol} / \mathrm{l}$. The mean MRT was 715.3 (SD 204.1) milliseconds. Overall,
$57.1 \%$ of participants had a subclinical magnesium deficiency. More than half of the participants were highly educated and $33.9 \%$ have low education levels. $67.3 \%$ of subjects were nonsmokers, $18.7 \%$ were smokers and $14 \%$ were ex-smokers. Overall, leisure-time physical activity was low. The majority of the sample was overweight $(38.2 \%)$ or obese $(32.5 \%)$. A total of $61.4 \%$ of the sample reported using supplements. $38.3 \%$ were using vitamin $\mathrm{D}$ and calcium supplements. Supplementary Figure S1 shows the distribution of serum magnesium concentrations among the study sample. Most of the participants had concentrations between $0.75-0.9 \mathrm{mmol} / \mathrm{l}$.

Across the quartiles of serum magnesium from the lowest to the highest, there was no difference in age, gender, education, and smoking (Table 1). However, insulin use, diabetes oral medication, and hypertension medication were higher among people who have low serum magnesium. Age and female gender were associated with a higher MRT while education was associated with a lower MRT (Supplementary Figure S2). 


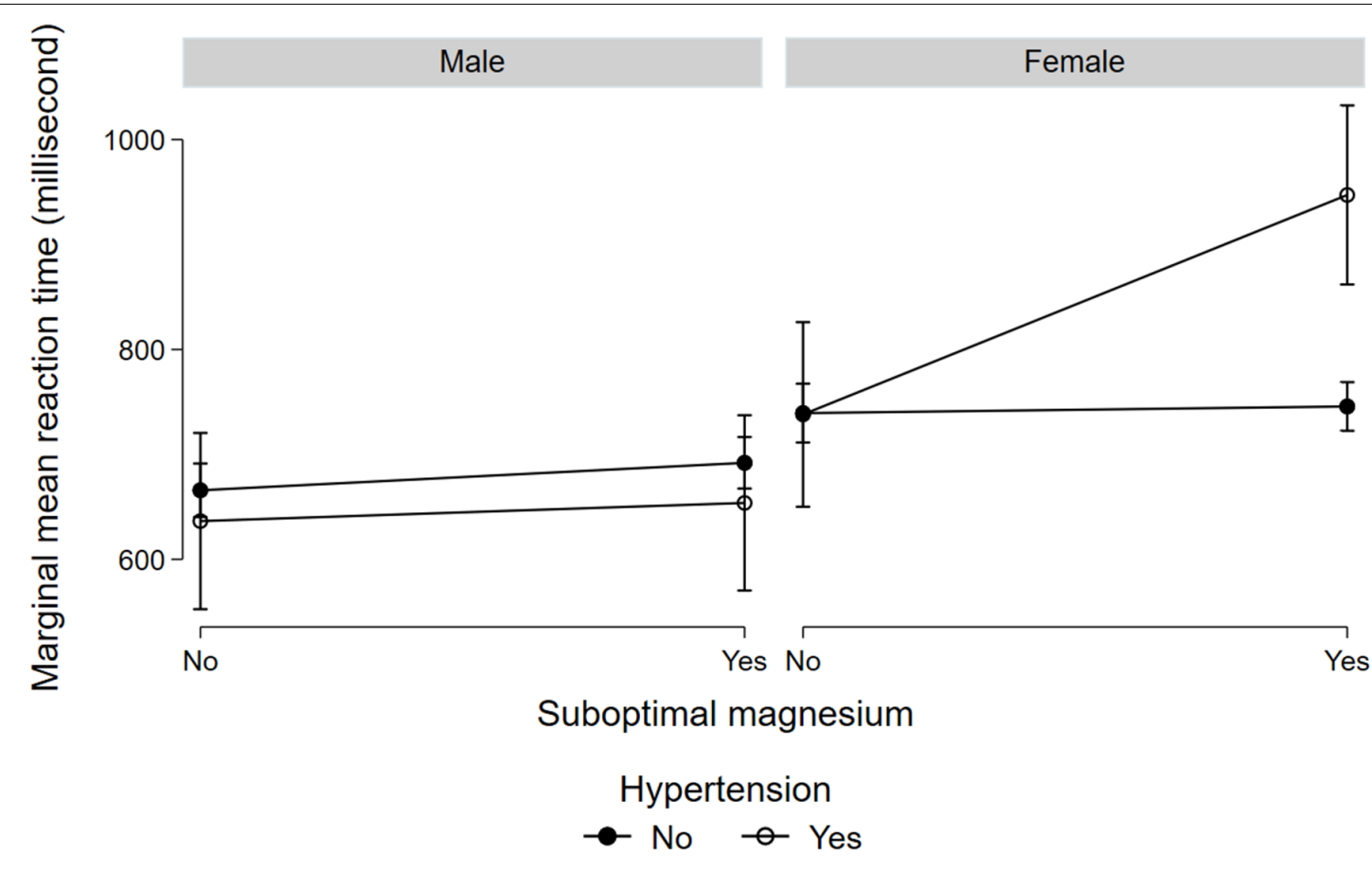

$\mathrm{p}$ for gender, hypertension and magnesium interaction 0.008

FIGURE 1 | Interaction between serum magnesium concentrations, hypertension, and gender with cognition. Values represent the adjusted mean (SD) of mean reaction time (MRT) derived from multivariable linear regression. Model adjusted for gender, age, education, BMl, smoking status, leisure-time physical activity, diabetes, hypertension, and current medication use for hypertension and diabetes.

No association was observed between MRT and smoking and physical activity.

\section{Association Between Serum Magnesium With MRT}

Serum magnesium concentration was inversely associated with MRT. After adjusting for sociodemographic characteristics, lifestyle factors, across the quartiles of serum magnesium, the regression coefficients (95\% CI) for MRT were 0 (reference), -17.8 (2nd quartile), -18.3 (3rd quartile), and -31.9 (4th quartile; $p$ for trend 0.05; Table 2). Further adjusting for hypertension, diabetes, and medication use, the above association was attenuated and became statistically nonsignificant. Similarly, using serum magnesium as a continuous variable, there was an inverse relationship between magnesium and MRT before the adjustment of medication use.

Figure 1 illustrated a significant interaction $(p=0.008)$ between gender, low serum magnesium, and hypertension in relation to MRT. Women with suboptimal magnesium and hypertension had the highest MRT. Although a three-way interaction between gender, suboptimal magnesium, and diabetes was not statistically significant $(p=0.173)$, women with suboptimal magnesium and diabetes had the highest MRT (Figure 2). There was a significant interaction between suboptimal magnesium, gender, and age in relation to MRT (Figure 3). Among women with suboptimal magnesium, there was an abrupt increase in the MRT when the age was above 40 years old. No such association was observed in men.

\section{DISCUSSION}

In this cross-sectional study, serum magnesium concentration was inversely associated with MRT. Low serum magnesium was prevalent in Qatari adults and was associated with longer MRT. There was a significant interaction between low serum magnesium, hypertension, and gender in relation to MRT. There was also an interaction between gender, diabetes, and low serum magnesium. Those with hypertension and low serum magnesium concentrations, the MRT was longer in women but not in men.

Our findings are consistent with previous studies on the association between serum magnesium concentrations and cognition in both animal and human studies (Allaert et al., 2016; Serefko et al., 2016; Balmus et al., 2017; Boyle et al., 2017; Iolascon et al., 2017; Kirkland et al., 2018). In a case-control study of 30 patients (15 with Alzheimer's disease and 15 with mild cognitive impairment) and 15 control participants, lower magnesium concentrations were found among patients with Alzheimer's disease and mild cognitive impairment (Balmus et al., 2017). Evidence from animal studies supports a link between circulating magnesium and cognition (Slutsky et al., 2010; Li et al., 2014; Huang et al., 2018; Tu et al., 2018). Low magnesium impairs cognition (Tu et al., 2018) and 

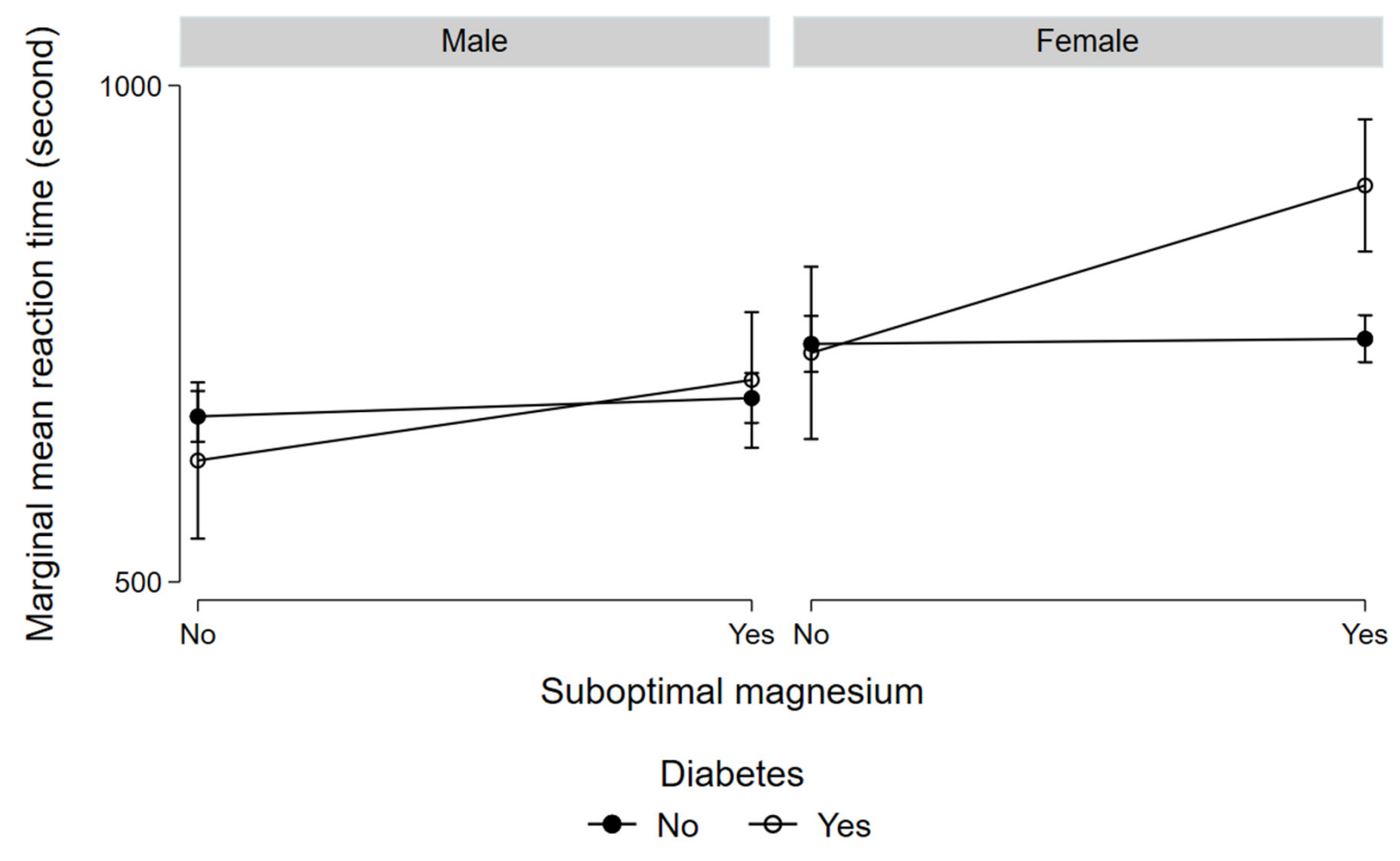

$p$ for gender, diabetes and magnesium interaction 0.173

FIGURE 2 | Interaction between serum magnesium concentrations, diabetes, and gender with cognition. Values represent the adjusted mean (SD) of MRT derived from multivariable linear regression. Model adjusted for gender, age, education, BMI, smoking status, leisure-time physical activity, diabetes, hypertension, and current medication use for hypertension and diabetes.

magnesium supplementation improves cognition in several studies (Slutsky et al., 2010; Li et al., 2014; Huang et al., 2018). For example, mice fed with a magnesium-deficient diet showed impairments in the three types of hippocampus-dependent memory including contextual, social recognition, and spatial memories (Noble et al., 2014). In contrast, rats with hepatic encephalopathy after administration of oral magnesium showed an improvement in cognitive and locomotor functions by reducing the brain manganese concentration and regulating glutamine synthetase (Han et al., 2019).

Human studies on the relationship between magnesium intake and cognition are limited (Iranpour et al., 2019). Data from the National Health and Nutrition Examination Survey (NHANES) showed that magnesium intake was directly associated with improved cognitive function markers such as Digital Symbol Substitution, Animal Fluency tests, immediate recall score, and delayed recall score (Iranpour et al., 2019). Diets rich in magnesium have shown to be positively associated with cognition (Shakersain et al., 2018). A study conducted in Sweden found that subjects who consumed a Nordic Prudent diet rich in magnesium were associated with an improved cognitive function (Shakersain et al., 2018). A prospective cohort study in New York $(n=2148)$ suggested that Alzheimer's Disease risk could be decreased with the adherence to a dietary pattern characterized by high consumption of magnesium-rich foods such as fish, vegetables, and fruits (Gu et al., 2010). Findings from clinical trials also support the beneficial effects of magnesium on cognition (Cohen-Hagai et al., 2018). In a recent prospective, randomized, double-blinded study $(n=22)$, a positive association between serum and intracellular magnesium and cognitive performance was observed. Also, magnesium deficiency was associated with impaired cognition (Cohen-Hagai et al., 2018). In a 12-week randomized, double-blind, placebocontrolled, parallel designed trial $(n=44)$, magnesium has been shown to have the potential for treating cognitive impairment (Liu et al., 2016).

The interaction between gender, hypertension/diabetes, and low serum magnesium is intriguing. Although the mechanisms are not clear, maintaining adequate circulating magnesium seems to be especially important in women. Consistent with previous studies (Hyde, 2016), in this study, women have lower MRT compared to men. Further studies are needed to elucidate the mechanisms linking magnesium with cognition. Further clinical trials are warranted to test the efficacy of magnesium supplementation in cognition in women with diabetes and hypertension.

Several mechanisms may explain the association between magnesium and cognition. First, adequate magnesium is essential for the prevention and management of diabetes and hypertension. Diabetes and hypertension (Tadic et al., 2016; 

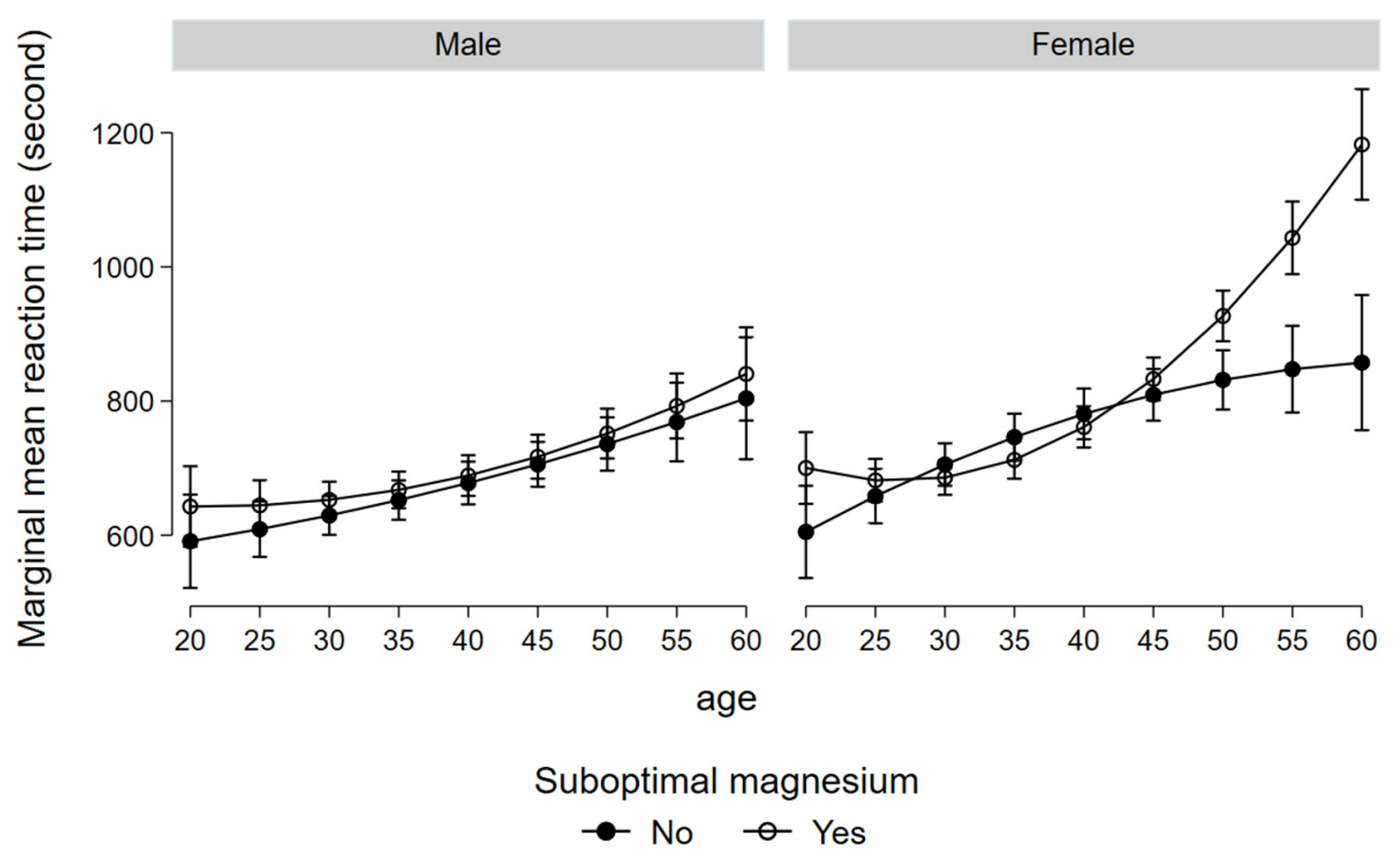

p for interation 0.009

FIGURE 3 | Interaction between gender, suboptimal serum magnesium, and age in relation to MRT. Values represent the adjusted mean (SD) of MRT derived from multivariate linear regression. Model adjusted for gender, age, education, BMI, smoking status, leisure-time physical activity, diabetes, hypertension, and current medication use for hypertension and diabetes.

Biessels and Despa, 2018) are risk factors for cognitive impairment. In Qatar, the diabetes burden is among one of the highest in the world (Al-Thani et al., 2017a). Second, magnesium has antioxidant properties and can reduce the levels of oxidative stress and inflammation (Zheltova et al., 2016). The latter plays an important role in brain aging and cognitive function decline (Walker et al., 2019).

The causes of low serum magnesium in this study may be multifactorial. Diabetes and hypertension can be significant contributors to hypomagnesemia. In Qatar, the prevalence of diabetes was $\approx 19 \%$ among adults aged $18-64$ years old (Al-Thani et al., 2017a). The contribution of diet on serum magnesium levels seems to be small based on a previous study in Qatar. A dietary pattern using a reduced rank regression method with serum magnesium as the response variable can only explain $0.9 \%$ of the variance of serum magnesium (Shi and AbouSamra, 2019). In Qatar, the main source of drinking water is desalinated seawater. Magnesium concentration in desalinated water is significantly lower than in non-desalinated municipal water. For example, desalinated water contained $10.9 \mathrm{mg} / \mathrm{l}$ compared to $18 \mathrm{mg} / \mathrm{l}$ in other bottled water (Rowell et al., 2015). It is not known to what extent the consumption of desalinated seawater contributes to the low serum magnesium in Qatar.

On the other hand, based on the dietary patterns in Qatar, refined cereals/grains were the second most consumed product in Qatar. Refined grains tend to be low in magnesium. Moreover, Qataris consume more oils, fats, sugar, and sweets than non-Qataris (Al-Thani et al., 2017b). This may also contribute to lower magnesium dietary intake. Further studies are needed to identify the contributors of serum magnesium. With the population aging rapidly, in combination with the high burden of chronic diseases, intervention is needed to prevent cognitive function decline.

Our study has several strengths. First, the participants were from the general population in Qatar. Second, because of the richness of $\mathrm{QBB}$ data, we were able to adjust the analysis for various potential confounding variables. Because of the cross-sectional study design, the results should not be viewed in terms of cause and effect. Another limitation was that the cognitive function test included only MRT. As QBB started to conduct an MRI brain scan, it will allow us to examine the relationship between serum magnesium and brain image in the future. Furthermore, the mean age of the sample was young. It may not represent the Qatar population at-large.

\section{CONCLUSION AND FUTURE WORK}

There was a direct association between serum magnesium and cognitive function. Low magnesium concentrations were associated with a longer MRT. Women with diabetes or 
hypertension were affected the greatest by low magnesium concentrations. Therefore, they may derive benefits from the consumption of magnesium-rich foods such as nuts, fish, leafy vegetables, and legumes. Further longitudinal studies and clinical trials are needed to verify whether magnesium intake and supplementation may improve cognitive function in the population.

\section{DATA AVAILABILITY STATEMENT}

The dataset generated for this study are available on request to the Qatar Biobank Study data management team.

\section{ETHICS STATEMENT}

The QBB study was reviewed and approved by the Institutional Review Board (IRB) from the Hamad Medical Corporation Ethics Committee. All participants gave written informed consent before participation. The current study was approved under the IRB exempted category (Ex-2019-RES-ACC-01630086) by the QBB.

\section{REFERENCES}

Al Kuwari, H., Al Thani, A., Al Marri, A., Al Kaabi, A., Abderrahim, H., Afifi, N., et al. (2015). The Qatar Biobank: background and methods. BMC Public Health 15:1208. doi: 10.1186/s12889-015-2522-7

Allaert, F. A., Courau, S., and Forestier, A. (2016). Effect of magnesium, probiotic, and vitamin food supplementation in healthy subjects with psychological stress and evaluation of a persistent effect after discontinuing intake. Panminerva Med. 58, 263-270.

Al-Thani, M., Al-Thani, A. A., Al-Chetachi, W., Khalifa, S. E., Vinodson, B., Al-Malki, B., et al. (2017a). Situation of diabetes and related factors among qatari adults: findings from a community-based survey. JMIR Diabetes 2:e7. doi: 10.2196/diabetes.7535

Al-Thani, M., Al-Thani, A. A., Al-Mahdi, N., Al-Kareem, H., Barakat, D., Al-Chetachi, W., et al. (2017b). An overview of food patterns and diet quality in qatar: findings from the national household income expenditure survey. Cureus 9:e1249. doi: 10.7759/cureus.1249

Al-Thani, A., Fthenou, E., Paparrodopoulos, S., Al Marri, A., Shi, Z., Qafoud, F., et al. (2019). Qatar biobank cohort study: study design and first results. Am. J. Epidemiol. 188, 1420-1433. doi: 10.1093/aje/kwz084

Alzheimer's Disase International. (2018). World Alzheimer Report 2018. London: Alzheimer's Disease International.

American Diabetes Association. (2014). Diagnosis and classification of diabetes mellitus. Diabetes Care 37, S81-S90. doi: 10.2337/dc14-S081

Azab, M., Al-Shudifat, A.-E., Agraib, L., Allehdan, S., and Tayyem, R. (2019). Does micronutrients intake modulate the risk of coronary heart disease? Nutr. Food Sci. 49, 368-380. doi: 10.1108/nfs-06-2018-0176

Balmus, I. M., Strungaru, S. A., Ciobica, A., Nicoara, M. N., Dobrin, R., Plavan, G., et al. (2017). Preliminary data on the interaction between some biometals and oxidative stress status in mild cognitive impairment and Alzheimer's disease patients. Oxid. Med. Cell. Longev. 2017:7156928. doi: 10.1155/2017/7156928

Barbagallo, M., Belvedere, M., Di Bella, G., and Dominguez, L. J. (2011). Altered ionized magnesium levels in mild-to-moderate Alzheimer's disease. Magnes. Res. 24, S115-S121. doi: 10.1684/mrh.2011.0287

Biessels, G. J., and Despa, F. (2018). Cognitive decline and dementia in diabetes mellitus: mechanisms and clinical implications. Nat. Rev. Endocrinol. 14, 591-604. doi: 10.1038/s41574-018-0048-7

Boyle, N. B., Lawton, C., and Dye, L. (2017). The effects of magnesium supplementation on subjective anxiety and stress-A systematic review. Nutrients 9:E429. doi: 10.3390/nu9050429

\section{AUTHOR CONTRIBUTIONS}

ZS: conceptualization, formal analysis, resources, supervision, and project administration. ZS, VG, KA-G, SE, AM, and TAA: methodology and writing-review and editing. ZS, VG, KAG, SE, AM, and TA-A: writing-original draft preparation. All authors read and approved the final version of the manuscript.

\section{ACKNOWLEDGMENTS}

We would like to thank the members and staff of Qatar Biobank for providing the data. We are grateful to all the Qatar Biobank participants. The publication of this article was funded by the Qatar National Library.

\section{SUPPLEMENTARY MATERIAL}

The Supplementary Material for this article can be found online at: https://www.frontiersin.org/articles/10.3389/fnagi.2020.001 01/full\#supplementary-material.

Cherbuin, N., Kumar, R., Sachdev, P. S., and Anstey, K. J. (2014). Dietary mineral intake and risk of mild cognitive impairment: the PATH through life project. Front. Aging Neurosci. 6:4. doi: 10.3389/fnagi.2014. 00004

Cohen-Hagai, K., Feldman, D., Turani-Feldman, T., Hadary, R., Lotan, S., and Kitay-Cohen, Y. (2018). Magnesium deficiency and minimal hepatic encephalopathy among patients with compensated liver cirrhosis. Isr. Med. Assoc. J. 20, 533-538.

Costello, R. B., Elin, R. J., Rosanoff, A., Wallace, T. C., Guerrero-Romero, F., Hruby, A., et al. (2016). Perspective: the case for an evidence-based reference interval for serum magnesium: the time has come. Adv. Nutr. 7, 977-993. doi: 10.3945/an.116.012765

Costello, R., Wallace, T. C., and Rosanoff, A. (2016). Magnesium. Adv. Nutr. 7, 199-201. doi: 10.3945/an.115.008524

Fang, X., Wang, K., Han, D., He, X., Wei, J., Zhao, L., et al. (2016). Dietary magnesium intake and the risk of cardiovascular disease, type 2 diabetes, and all-cause mortality: a dose-response meta-analysis of prospective cohort studies. BMC Med. 14:210. doi: 10.1186/s12916-016-0742-z

Gröber, U., Schmidt, J., and Kisters, K. (2015). Magnesium in prevention and therapy. Nutrients 7, 8199-8226. doi: 10.3390/nu7095388

Gu, Y., Nieves, J. W., Stern, Y., Luchsinger, J. A., and Scarmeas, N. (2010). Food combination and Alzheimer disease risk: a protective diet. Arch. Neurol. 67, 699-706. doi: 10.1001/archneurol.2010.84

Han, J., Plummer, J., Liu, L., Byrd, A., Aschner, M., and Erikson, K. M. (2019). The impact of obesity on brain iron levels and $\alpha$-synuclein expression is regionally dependent. Nutr. Neurosci. 22, 335-343. doi: 10.1080/1028415X.2017. 1387720

Huang, Y., Huang, X., Zhang, L., Han, F., Pang, K. L., Li, X., et al. (2018). Magnesium boosts the memory restorative effect of environmental enrichment in Alzheimer's disease mice. CNS Neurosci. Ther. 24, 70-79. doi: 10.1111/cns. 12775

Hugo, J., and Ganguli, M. (2014). Dementia and cognitive impairment: epidemiology, diagnosis, and treatment. Clin. Geriatr. Med. 30, 421-442. doi: 10.1016/j.cger.2014.04.001

Hyde, J. S. (2016). Sex and cognition: gender and cognitive functions. Curr. Opin. Neurobiol. 38, 53-56. doi: 10.1016/j.conb.2016.02.007

Iolascon, G., Gimigliano, R., Bianco, M., De Sire, A., Moretti, A., Giusti, A., et al. (2017). Are dietary supplements and nutraceuticals effective for musculoskeletal health and cognitive function? A scoping review. J. Nutr. Health Aging 21, 527-538. doi: 10.1007/s12603-016-0823-x 
Iranpour, S., Saadati, H. M., Koohi, F., and Sabour, S. (2019). Association between caffeine intake and cognitive function in adults; effect modification by sex: data from National Health and Nutrition Examination Survey (NHANES) 2013-2014. Clin. Nutr. doi: 10.1016/j.clnu.2019.09.003 [Epub ahead of print].

Jahnen-Dechent, W., and Ketteler, M. (2012). Magnesium basics. Clin. Kidney J. 5 , i3-i14. doi: 10.1093/ndtplus/sfr163

Kirkland, A. E., Sarlo, G. L., and Holton, K. F. (2018). The role of magnesium in neurological disorders. Nutrients 10:E730. doi: 10.3390/nu10 060730

Li, W., Yu, J., Liu, Y., Huang, X., Abumaria, N., Zhu, Y., et al. (2014). Elevation of brain magnesium prevents synaptic loss and reverses cognitive deficits in Alzheimer's disease mouse model. Mol. Brain 7:65. doi: 10.1186/s13041-0140065-y

Liu, G., Weinger, J. G., Lu, Z. L., Xue, F., and Sadeghpour, S. (2016). Efficacy and safety of MMFS-01, a synapse density enhancer, for treating cognitive impairment in older adults: a randomized, double-blind, placebo-controlled trial. J. Alzheimers Dis. 49, 971-990. doi: 10.3233/jad-150538

Lyall, D. M., Cullen, B., Allerhand, M., Smith, D. J., Mackay, D., Evans, J., et al. (2016). Cognitive test scores in UK biobank: data reduction in 480,416 participants and longitudinal stability in 20,346 participants. PLoS One 11:e0154222. doi: 10.1371/journal.pone.0154222

Noble, E. E., Mavanji, V., Little, M. R., Billington, C. J., Kotz, C. M., and Wang, C. (2014). Exercise reduces diet-induced cognitive decline and increases hippocampal brain-derived neurotrophic factor in CA3 neurons. Neurobiol. Learn. Mem. 114, 40-50. doi: 10.1016/j.nlm.2014.04.006

Rowell, C., Kuiper, N., and Shomar, B. (2015). Potential health impacts of consuming desalinated bottled water. J. Water Health 13, 437-445. doi: $10.2166 /$ wh. 2014.128

Scarmeas, N., Anastasiou, C. A., and Yannakoulia, M. (2018). Nutrition and prevention of cognitive impairment. Lancet Neurol. 17, 1006-1015. doi: 10.1016/S1474-4422(18)30338-7

Serefko, A., Szopa, A., and Poleszak, E. (2016). Magnesium and depression. Magnes. Res. 29, 112-119. doi: 10.1684/mrh.2016.0407

Shakersain, B., Rizzuto, D., Larsson, S. C., Faxen-Irving, G., Fratiglioni, L., and $\mathrm{Xu}, \mathrm{W}$. L. (2018). The nordic prudent diet reduces risk of cognitive decline in the swedish older adults: a population-based cohort study. Nutrients 10:E229. doi: $10.3390 /$ nu10020229
Shi, Z., and Abou-Samra, A. B. (2019). Association of low serum magnesium with diabetes and hypertension: findings from Qatar Biobank Study. Diabetes Res. Clin. Pract. 158:107903. doi: 10.1016/j.diabres.2019. 107903

Slutsky, I., Abumaria, N., Wu, L. J., Huang, C., Zhang, L., Li, B., et al. (2010). Enhancement of learning and memory by elevating brain magnesium. Neuron 65, 165-177. doi: 10.1016/j.neuron.2009.12.026

Tadic, M., Cuspidi, C., and Hering, D. (2016). Hypertension and cognitive dysfunction in elderly: blood pressure management for this global burden. BMC Cardiovasc. Disord. 16:208. doi: 10.1186/s12872-016-0386-0

Tu, X., Qiu, H., Lin, S., He, W., Huang, G., Zhang, X., et al. (2018). Low levels of serum magnesium are associated with poststroke cognitive impairment in ischemic stroke patients. Neuropsychiatr. Dis. Treat. 14, 2947-2954. doi: $10.2147 /$ ndt.s181948

Walker, K. A., Gottesman, R. F., Wu, A., Knopman, D. S., Gross, A. L., Mosley, T. H. Jr., et al. (2019). Systemic inflammation during midlife and cognitive change over 20 years: the ARIC study. Neurology 92, e1256-e1267. doi: 10.1212/WNL.0000000000007094

Xu, Z. P., Li, L., Bao, J., Wang, Z. H., Zeng, J., Liu, E. J., et al. (2014). Magnesium protects cognitive functions and synaptic plasticity in streptozotocin-induced sporadic Alzheimer's model. PLoS One 9:e108645. doi: 10.1371/journal.pone. 0108645

Zheltova, A. A., Kharitonova, M. V., Iezhitsa, I. N., and Spasov, A. A. (2016). Magnesium deficiency and oxidative stress: an update. Biomedicine Taipei 6:20. doi: 10.7603/s40681-016-0020-6

Conflict of Interest: The authors declare that the research was conducted in the absence of any commercial or financial relationships that could be construed as a potential conflict of interest.

Copyright (C) 2020 Al-Ghazali, Eltayeb, Musleh, Al-Abdi, Ganji and Shi. This is an open-access article distributed under the terms of the Creative Commons Attribution License (CC BY). The use, distribution or reproduction in other forums is permitted, provided the original author(s) and the copyright owner(s) are credited and that the original publication in this journal is cited, in accordance with accepted academic practice. No use, distribution or reproduction is permitted which does not comply with these terms. 